\title{
A Clinicopathological Study of Thrombocytopenia in Malaria Cases with Its Evaluation in Different Types of Malaria
}

\author{
Nisha Thattamparambil Gopalakrishnan ${ }^{1}$, Supriya Papaiah², Smera Soman³ ${ }^{3}$ Krishnaraj Upadhyaya4 \\ ${ }^{1}$ Department of Pathology, MES Medical College, Perinthalmanna, Malappuram, Kerala, India. ${ }^{2,3,4}$ Department of \\ Pathology, Yenepoya Medical College, Yenepoya (Deemed to Be University), Mangalore, Karnataka, India.
}

\section{ABSTRACT}

\section{BACKGROUND}

Malaria is a global health problem, caused by the protozoa plasmodium and is characterized by haematological abnormalities, with thrombocytopenia being the most common. Microscopic examination of thick and thin blood films is the gold standard in diagnosis of malaria. This study was conducted to assess the severity of thrombocytopenia in malaria patients and to correlate it with the type of malaria.

\section{METHODS}

A retrospective study was conducted in Yenepoya Medical College, Hospital, Mangalore for a period of 1.5 years. Patients of all ages who were hospitalized or attending OPD were included. Patients with dengue fever and drug-induced thrombocytopenia were excluded. Complete blood cell count was done using an automated cell count analyser. Thrombocytopenia was defined as a platelet count < $150,000 / \mu \mathrm{l}$. It was graded as severe: platelet count $<50,000 / \mu \mathrm{l}$, moderate: 50,000 100,000/ $\mu$ l and mild: 100,000-150,000/ $\mu \mathrm{l}$.

\section{RESULTS}

Our study included 120 malaria positive cases with 102 (85\%) males and 18 (15 $\%$ ) females. $90.8 \%$ cases presented with thrombocytopenia, predominantly moderate to severe thrombocytopenia $(80.7 \%)$. Plasmodium vivax (Pv) was the most common species found in our study. Ninety-nine (82.5\%) cases were positive for Plasmodium vivax (Pv), 8 (6.6\%) cases for Plasmodium falciparum (Pf) and 13 $(10.8 \%)$ cases had mixed infection with both Plasmodium vivax and Plasmodium falciparum. Out of 99 cases which had vivax malaria, 88 (88.9\%) cases had thrombocytopenia. All 8 cases detected with falciparum malaria and 13 cases with mixed infection had thrombocytopenia.

\section{CONCLUSIONS}

The above findings can have therapeutic implications in avoiding unnecessary platelet infusion in malaria patients. Presence of thrombocytopenia in a patient with acute febrile illness can heighten suspicion of malaria, and initiate prompt treatment.

\section{KEY WORDS}

Thrombocytopenia, Malaria, Severity
Corresponding Author: Dr. Supriya Papaiah, Department of Pathology, Yenepoya Medical College, Yenepoya (Deemed to be University), Mangalore - 575018, Karnataka, India. E-mail: supriya.papaiah@gmail.com

\section{DOI: $10.14260 /$ jemds/2021/553}

How to Cite This Article: Gopalakrishnan NT, Papaiah S, Soman S. et al. A clinicopathological study of thrombocytopenia in malaria cases with its evaluation in different types of malaria. $J$ Evolution Med Dent Sci 2021;10(33): 2707-2711, DOI: 10.14260/jemds/2021/553

Submission 08-04-2021, Peer Review 17-06-2021, Acceptance 22-06-2021, Published 16-08-2021.

Copyright (C) 2021 Nisha Thattamparambil Gopalakrishnan et al. This is an open access article distributed under Creative Commons Attribution License [Attribution 4.0 International (CC BY 4.0)] 


\section{BACKGROUND}

Malaria is a global health problem with increased morbidity and mortality and an annual incidence of about 216 million cases and an estimated 6,55,000 deaths in 2010.1,2 It is endemic in several countries and India contributes about $77 \%$ of the total malaria cases in Southeast Asia.2,3 Malaria is caused by infection with plasmodium species and transmitted to people by infected female Anopheles mosquito. Five species of plasmodium which cause malaria in humans are Plasmodium vivax, Plasmodium falciparum, Plasmodium malariae, Plasmodium ovale, and Plasmodium knowlesi.1,2,3,4 In India about $27 \%$ population live in malaria high transmission area and $58 \%$ in low transmission area. Among the overall 1.52 million cases of malaria, 0.76 million cases were due to Plasmodium falciparum, accounting to $50 \%$ of the cases and 924 deaths from malaria. 4,5,6,7 Three distinct clinical stages in malarial infection are cold stage, hot stage, and sweating stage. The clinical features of malaria vary from mild to severe, and complicated, according to the patient's immunity state, the intensity of infection and also the presence of other co-morbid conditions. ${ }^{4}$ Since malaria parasite affects multiple organs of the body like spleen, liver, brain, gastrointestinal tract and blood vessels, there is wide spectrum of clinical picture ranging from simple malaise to life threatening central nervous system complications like coma.4,8,9

The gold standard in the diagnosis of malaria is examination of thick and thin blood films under the light microscope. ${ }^{2}$ Malarial antigen-based rapid diagnostic tests are a valid alternative to microscopy, but they are expensive. Haematological abnormalities like anaemia and thrombocytopenia, have been observed in malaria patients with thrombocytopenia (TP) being the most common. ${ }^{2,4,8,9}$ Although thrombocytopenia is a reliable diagnostic marker, prognostic implications could vary in different types of malaria. A finding of thrombocytopenia should raise the suspicion of malaria thus prompting a more diligent search for the malaria parasite and perform more specific tests, including multiple peripheral blood smears and ELISA for parasite-specific antigen.2,4,6 Various immunopathological studies have shown that platelets are an important component of the host innate immune responses against malaria infection. ${ }^{10,11,12}$ A number of observational studies also have confirmed the association of thrombocytopenia to malaria, with both non-immunological as well as immunological destruction of platelets being implicated in causing thrombocytopenia. ${ }^{13}$ Low platelet counts are commonly encountered in malaria types, and platelet values less than $60,000 / \mu \mathrm{L}$ have been reported in $29-46 \%$ of patients affected with Plasmodium vivax malaria. However, thrombocytopenia is not considered as a severity biomarker in Plasmodium falciparum malaria, and it has not yet been validated as an independent severity parameter in Plasmodium vivax malaria. Numerous studies and case reports have shown that severe thrombocytopenia was described as the most important severity sign of vivax malaria. Therefore, it cannot be excluded that the use of such an indicator may lead to an overestimation of Plasmodium vivax malaria severity. ${ }^{14}$ The speculated mechanisms which can cause thrombocytopenia are sequestration in spleen, coagulation disorders, oxidative stress and antibody mediated platelet destruction. ${ }^{4,15,16}$ Since there is a paucity of data in studies done on correlation of presence and severity of thrombocytopenia with the type of malaria in Indian studies, this study was conducted to assess the severity of thrombocytopenia in malaria patients and to correlate it with the type of malaria.

\section{METHODS}

A retrospective study was conducted for a period of one year and five months from August 2017 to December 2018 in a tertiary care hospital attached to a medical college, Dakshina Kannada district. A total of 120 patients who were positive for malaria were included in the study. Malaria test was carried out by thin peripheral blood smear examination and stained by Leishman stain. Patients who were diagnosed with dengue fever and drug-induced thrombocytopenia were excluded from the study. Complete blood cell count was done using an automated cell count analyser (SysmexXN1000). Platelet count of less than $150,000 / \mu \mathrm{l}$ was used to define thrombocytopenia. Patients were divided into three subgroups based on the platelet count. Thrombocytopenia was graded as severe if platelet count was less than 50,000 / $\mu \mathrm{l}$, moderate if 50,000 - 100,000 / $\mu \mathrm{l}$ and mild if 100,000$150,000 / \mu l^{2}$ Data was entered into an Excel sheet and statistical analysis was performed using SPSS version 23 .

\section{RESULTS}

In our study, 120 patients with malaria positivity were investigated with platelet count. Almost all cases 120 (100\%) presented with fever as the chief complaint associated with chills and rigors, followed by fatigue (90\%), myalgia (65\%), vomiting (60\%), anorexia (70\%) and headache (35\%). On clinical examination, splenomegaly was seen in about $20 \%$ cases and hepatomegaly in $1 \%$ cases. The age of the patients ranged from 9 years to 69 years with majority of the patients in the age group of 21-30 years (31.6\%). Among the 120 cases, 102 (85\%) patients were males and 18 (15\%) patients were females with male to female ratio of $5: 1$. Distribution of cases for different age groups is given in table 1.

\begin{tabular}{|ccc|}
\hline Age & Distribution & Percentage (\%) \\
\hline 1-10 years & 3 & 2.5 \\
11-20 years & 24 & 20 \\
21-30 years & 38 & 31.6 \\
31-40 years & 18 & 15 \\
41-50 years & 26 & 21.6 \\
51-60 years & 7 & 5.8 \\
61-70 years & 4 & 3.3 \\
Total no of Cases & $\mathbf{1 2 0}$ & \\
\hline Table 1. Distribution of Cases in Different Age Groups \\
\hline
\end{tabular}

Of the 120 malaria cases, thrombocytopenia was noted in $109(90.8 \%)$ cases and platelet count was normal in $11(9.2$ $\%$ ) cases. Mild thrombocytopenia was noted in 21 (19.3\%) cases, moderate in 36 (33\%) cases and severe thrombocytopenia in 52 (47.7\%) cases. Ninety-nine (82.5\%) cases were positive for Plasmodium vivax (Pv), 8 (6.6\%) 
cases for Plasmodium falciparum (Pf) and 13 (10.8\%) cases had mixed infection. Thus, Plasmodium vivax was the most common malaria species found in our area of study. While analysing the severity of malaria, out of 99 cases of Plasmodium vivax infection, majority of 48 cases had severe $(3+$ and $4+)$ infection and 35 cases had moderate infection $(2+)$ and 16 cases had mild $(1+)$ infection. Whereas out of 8 cases of Plasmodium falciparum, 5 cases had mild infection, 2 had moderate infection and only 1 case had severe infection. Out of 13 cases with mixed infection, 8 cases showed only mild $(1+)$ infection and 5 cases showed moderate to severe $(2+, 3+)$ infection.

The mean platelet count in Plasmodium vivax malaria was $57,124 / \mu \mathrm{l}$ with a range of 13,000 to $4,40,000 / \mu \mathrm{l}$. Out of the 99 cases which had vivax malaria, 88 (88.9\%) cases had thrombocytopenia, 11 (11.1\%) cases had normal platelet count. Of these 88 cases, 29 (32.9\%) cases had severe thrombocytopenia, 41 (46.6\%) cases had moderate thrombocytopenia and 18 (20.5 \%) cases had mild thrombocytopenia.

The mean platelet count of Plasmodium falciparum was $59,074 / \mu$ l with a range of 27,000 to 108000 / $\mu$ l. All 8 cases detected with falciparum malaria, had thrombocytopenia. Out of these 8 cases, 3 (37.5\%) cases had severe, 4 (50\%) cases had moderate, and one (12.5\%) case had mild thrombocytopenia respectively.

In mixed infection, mean platelet count was $56,205 / \mu \mathrm{l}$ and range being 27,000 to $116000 / \mu$ l. Among the 13 cases of mixed infection, all cases had thrombocytopenia, out of which $4(30.8 \%)$ cases had severe thrombocytopenia, 7 (53.8\%) cases had moderate thrombocytopenia and 2 (15.4\%) cases had mild thrombocytopenia. Distribution of platelet count in different types of malaria is shown in table 2 .

\begin{tabular}{|c|c|c|c|c|c|}
\hline $\begin{array}{c}\text { Platelet } \\
\text { Count }\end{array}$ & $\begin{array}{c}<50,000 \\
/ \mu l\end{array}$ & $\begin{array}{c}51,000- \\
99,000 / \mu l\end{array}$ & $\begin{array}{c}100,000- \\
149,000 / \mu 1 .\end{array}$ & $\begin{array}{l}>1.5 \\
\text { lakhs }\end{array}$ & Total \\
\hline P. vivax & $29(29.3 \%)$ & $41(41.4 \%)$ & 18 (18.2 \%) & $11(11.1 \%)$ & ) 99 (82.5\%) \\
\hline P. falcip & & & & - & \\
\hline Mixed in & $4(30.8 \%)$ & 7 (53.8\%) & $2(15.4 \%)$ & - & $13(10.8 \%)$ \\
\hline Total & 36 & 52 & 21 & 11 & 120 \\
\hline
\end{tabular}

\section{DISCUSSION}

Malaria is a disease of global importance and World Health Organization (WHO) has reported a worldwide annual incidence of 247 million cases and malarial mortality of one million per year. ${ }^{17}$ Malaria is a common protozoal disease caused by infection with parasite genus plasmodium and remains one of the major health problems in the tropics and associated with increased morbidity and mortality.2,4 Malaria affects almost all the blood components with thrombocytopenia and anaemia being often associated with it. ${ }^{4}$ Thrombocytopenia in malaria is usually of mild to moderate degree and rarely accompanied by clinical bleeding or disseminated intravascular coagulation (DIC).2,4,18 Malaria is a true haematological infectious disease with anaemia and thrombocytopenia being the most frequent malariaassociated haematological complications, and these have received more attention in the scientific literature due to their associated mortality.10,11,12 The mechanism of thrombocytopenia in malaria is not fully understood and it is thought to be a multifactorial phenomenon. ${ }^{10,11}$

However various studies have hypothesized the possible pathoimmunological mechanisms of thrombocytopenia in malaria. Immunoglobulin $\mathrm{G}$ binding to platelet-bound malaria antigens, oxidative stress, macrophage colony stimulating factor, spleen pooling, platelet phagocytosis and increased plasma cell free circulating nucleic acid levels in Plasmodium vivax are possible mechanisms resulting in damage to thrombocytes or resulting in excessive removal of platelets. ${ }^{10}$

Recently, Coelho et al. in their study demonstrated that macrophage-driven phagocytosis of platelets may be an important contributory mechanism and that the mean platelet volume was greater in thrombocytopenic patients with Plasmodium vivax malaria than in controls. The latter finding is particularly important and interesting because the presence of large circulating platelets may be viewed as compensatory mechanism in order to preserve primary haemostasis. Accordingly, bleeding is less commonly seen in the course of malaria even among patients with severe thrombocytopenia. ${ }^{14}$

Thrombocytopenia is a feature commonly seen in Plasmodium vivax and Plasmodium falciparum, regardless of the severity of infection. ${ }^{2,4}$ Studies have also shown that thrombocytopenia is so characteristic of malaria that in some places it is used as an indicator of malaria in patients presenting with fever.4,18,19,20 Hence patients with acute febrile illness with anaemia and thrombocytopenia without localizing signs should always alert the treating doctor about the possibility of malaria infection. ${ }^{2}$

In the present study, majority of the patients belonged to age group of 21 - 30 years (31.6\%) similar to the study done by Gupta et al. who found about $56 \%$ of the patients in the age group of 15 - 40 years. ${ }^{4}$ Whereas, in the study done by Gill et al. the maximum number of patients (43.33\%) were under the age of 20 years. ${ }^{2}$

Male preponderance was observed in our present study with a male to female ratio of $5: 1$, comparable to the study done by Gill et al. who found that 76 (63.33\%) were males and $44(36.66 \%)$ patients were females. ${ }^{2} \mathrm{~A}$ study of 230 patients by Gupta et al. also showed that, 150 cases (65.22\%) were males and 80 cases ( $34.78 \%$ ) were females. ${ }^{4}$

The commonest presenting clinical feature in our study was fever with chills and rigors followed by fatigue and myalgia. Similar observation was also seen in study done by Gill et al. who found that among 120 patients with malaria positivity investigated with platelet count, fever with chills and rigors was the most common presenting manifestation followed by headache and myalgia. ${ }^{2}$ Gupta et al. in his study also showed that all 230 cases (100\%) presented with fever, followed by weakness, nausea, vomiting, anorexia and diarrhoea. ${ }^{4}$ In our study, $90.8 \%$ patients with malaria presented with thrombocytopenia which is comparable with studies done by Kumar et al. and George et al. who found 88.8 $\%$ and $93.3 \%$ patients with thrombocytopenia respectively. ${ }^{21,22}$ (Table 3). Plasmodium vivax was the common malaria species seen in our study, similar to the finding in a study done by Gill et al. who found that 92 patients were positive for Plasmodium vivax malaria among the total 120 cases. $^{2}$ 
In the study done by Gupta et al. also showed that 56.51 $\%$ of patients were positive for Plasmodium vivax malaria. ${ }^{4}$ Similar observations was also seen in majority of the studies done by Faseela et al. Mohapatra et al. and Jadhav et al. 15,23,24,25,26 However in the studies done by Prasad et al. and Murthy et al. found, Plasmodium falciparum as the common malaria species. ${ }^{27,28}$

In the present study, 109 cases showed thrombocytopenia, out of which, mild thrombocytopenia was noted in $19.3 \%$ cases, moderate in $33 \%$ and severe thrombocytopenia in $47.7 \%$ cases respectively. Out of the 88 vivax cases, $32.9 \%$ cases had severe thrombocytopenia, 46.6 $\%$ cases had moderate thrombocytopenia and $20.5 \%$ cases had mild thrombocytopenia. Among the eight cases detected with falciparum malaria, $37.5 \%$ cases had severe, $50 \%$ cases had moderate and $12.5 \%$ cases had mild thrombocytopenia respectively. In the 13 cases of mixed infection, $30.8 \%$ cases had severe thrombocytopenia, $53.8 \%$ cases had moderate thrombocytopenia and $15.4 \%$ cases had mild thrombocytopenia. Similar observations were also seen in the study done by Gupta et al. who found that among 130 cases detected with vivax malaria, $8.69 \%$ cases had Grade I , 10.86 \% cases had Grade II, $17.39 \%$ cases had Grade III and $6.51 \%$ cases had Grade IV thrombocytopenia respectively and out of the 90 falciparum malaria cases, 15 (6.51\%) cases had Grade I thrombocytopenia, 20 (8.69\%) cases had Grade II, 35 $(15.17 \%)$ cases had Grade III and no patient was detected with Grade IV thrombocytopenia. Among 10 cases of mixed infection, $3(1.30 \%)$ cases had Grade I thrombocytopenia, 2 cases had Grade II, 3 (1.30 \%) cases had Grade III and 1 (0.43 $\%)$ case had Grade IV thrombocytopenia. 4

Numerous studies have shown that thrombocytopenia is a common manifestation equally observed in both Plasmodium vivax and falciparum malaria infections. A study review restricted to Brazil reported that the mortality rate in Plasmodium vivax malaria with severe thrombocytopenia alone was comparable to that of Plasmodium falciparum malaria. Study done by Naing $\mathrm{C}$ et al. suggested that magnitude of severe thrombocytopenia and mortality risks are similar in both Plasmodium vivax and Plasmodium falciparum malaria. ${ }^{10}$

The mean platelet count in Plasmodium vivax malaria in our study was $57,124 / \mu \mathrm{l}$ with a range of 13,000 to $4,40,000$ $/ \mu \mathrm{l}$ and the mean platelet count of Plasmodium falciparum was $59,074 / \mu \mathrm{l}$ with a range of 27,000 to $108000 / \mu \mathrm{l}$. Whereas study done by Gill et al. found the mean platelet count in Plasmodium vivax malaria as $1,27,652$ / $\mu \mathrm{l}$ with a range of $8000-3,50,000 / \mu \mathrm{l}$ as against Plasmodium falciparum malaria where the mean platelet count was $78,500 / \mu \mathrm{l}$ with a range of $9000-1,90,000 / \mu .^{2}$

In our study group of 120 patients with malaria infection, platelet count ranging from 50,000 / $\mu \mathrm{l}-1,50000 / \mu \mathrm{l}$ was noted in $88.9 \%$ cases of plasmodium vivax malaria as against $100 \%$ of cases of Plasmodium falciparum. Platelet count less than 50,000 / $\mu \mathrm{l}$ was noted in $29.3 \%$ cases of Plasmodium vivax as against $37.5 \%$ cases of Plasmodium falciparum malaria. Study done by Gill et al. showed that platelet count ranging from $50,000 / \mu \mathrm{l}-1,50000 / \mu \mathrm{l}$ was noted in $37 \%$ cases of Plasmodium vivax malaria as against $55.5 \%$ cases of Plasmodium falciparum. Platelet count less than 50,000 / $\mu \mathrm{l}$ was noted in only $17.4 \%$ cases of Plasmodium vivax malaria as against $33.3 \%$ cases of Plasmodium falciparum malaria. ${ }^{2}$

Presence of thrombocytopenia is not a distinguishing feature between falciparum and vivax malaria. ${ }^{2}$ It is usually believed that thrombocytopenia is more common in Plasmodium falciparum malaria, but contrary to the popular belief, Plasmodium vivax can also give rise to thrombocytopenia as seen in our study.

Similar observations were also seen in studies done by Gill et al. and Gupta et al.2,4 Recent studies from the Indian subcontinent, Qatar and Venezuela have found significant thrombocytopenia in Plasmodium vivax malaria than Plasmodium falciparum. ${ }^{4,23,24,29,30}$

\begin{tabular}{|cccc|}
\hline Various Studies & $\begin{array}{c}\text { Common } \\
\text { Malaria Species }\end{array}$ & $\begin{array}{c}\text { Number of } \\
\text { Cases }\end{array}$ & $\begin{array}{c}\text { Thrombocytopenia } \\
\text { (\%) }\end{array}$ \\
\hline Kumar et al..$^{21}$ & P. vivax & 27 & 88.8 \\
George et al. ${ }^{22}$ & P. vivax & 30 & 93.3 \\
Gill et al. & P. vivax & 120 & 63.3 \\
Prasad et al. & P. falciparum & 40 & 85 \\
Present study & P. vivax & 120 & 90.8 \\
\hline Table 3: Review of Various Studies, Estimating the Common Species \\
and Thrombocytopenia in Malaria Patients \\
\hline
\end{tabular}

The mechanism of thrombocytopenia in malaria is not known clearly. Immune-mediated lysis, sequestration in spleen and dyspoietic process in the bone marrow with diminished platelet production, have been postulated as mechanisms of thrombocytopenia in malaria.2,9 Platelet structural and functional abnormality have been demonstrated as a consequence of malaria. ${ }^{2}$ In the study done by Fajardo and Tallent in 1974 demonstrated the presence of Plasmodium vivax within platelets by electron microscopy and suggested a direct lytic effect of the parasite on the platelets. ${ }^{2,31}$

In the study done by Makkar RP et al. showed both nonimmunological destruction and immune mechanism are involving specific platelet-associated IgG antibodies that bind directly to the malarial antigen in the platelets that have been recently reported to play a role in the lysis of platelets and the development of thrombocytopenia.4,15,32

\section{CONCLUSIONS}

Patients with acute febrile illness and presence of thrombocytopenia, increases the possibility of malaria. In patients diagnosed with malaria, higher frequency of moderate to severe thrombocytopenia was observed. Thrombocytopenia is a most important feature in the laboratory diagnosis of malaria and its presence cannot distinguish between Plasmodium falciparum and Plasmodium vivax malaria.

Malaria infection has to be ruled out, if thrombocytopenia is present in any febrile illness, so as to avoid unnecessary expensive tests, and also correct diagnosis can be made to initiate prompt treatment.

Data sharing statement provided by the authors is available with the full text of this article at jemds.com.

Financial or other competing interests: None.

Disclosure forms provided by the authors are available with the full text of this article at jemds.com. 


\section{REFERENCES}

[1] World Health Organization, 2011, Malaria fact sheet.

[2] Gill MK, Makkar M, Bhat S, et al. Thrombocytopenia in malaria and its correlation with different types of malaria. Annals of Tropical Medicine and Public Health 2013;6(2):197.

[3] Khan SA, Ali W, Shahmi SN, et al. Platelet count in malaria. Pak J Pathol 2008;19(3):186-8.

[4] Gupta NK, Bansal SB, Jain UC, et al. Study of thrombocytopenia in patients of malaria. Tropical Parasitology 2013;3(1):58-61.

[5] WHO. World Malaria Report. Geneva Switzerland: WHO Press 2009.

[6] WHO. World Malaria Report. Geneva Switzerland: WHO Press 2008.

[7] Strategic Action Plan for Malaria Control in India 20072012, New Delhi: DGHS, Ministry of Health and Family Welfare, Government of India. 2009.

[8] Wickramasinghe SN, Abdalla SH. Blood and bone marrow changes in malaria. Baillieres Best Pract Res Clin Haematol 2000;13(2):277-99.

[9] Khan SJ, Khan FR, Usman M, et al. Malaria can lead to thrombocytopenia. Rawal Med J 2008;33(2):183-5.

[10] Naing C, Whittaker MA. Severe thrombocytopaenia in patients with vivax malaria compared to falciparum malaria: a systematic review and meta-analysis. Infectious Diseases of Poverty 2018;7(1):10.

[11] McMorran BJ, Marshall VM, De Graaf C, et al. Platelets kill intraerythrocytic malarial parasites and mediate survival to infection. Science 2009;323(5915):797-800.

[12] McMorran BJ, Burgio G, Foote SJ. New insights into the protective power of platelets in malaria infection. Commun Integer Biol 2013;6(3):e23653.

[13] Khan SJ, Abbass Y, Marwat MA. Thrombocytopenia as an indicator of malaria in adult population. Malaria Research and Treatment 2012;2012:405981.

[14] Antinori S, Corona A, Ridolfo AL, et al. Imported Plasmodium vivax malaria with severe thrombocytopaenia: can it be severe malaria or not? Malaria Journal 2016;15(1):1-9.

[15] Jadhav UM, Patkar VS, Kadam NN. Thrombocytopenia in malaria: correlation with type and severity of malaria. J Assoc Physicians India 2004;52:615-8.

[16] Rasheed A, Saeed S, Khan SA. Platelet count in malaria. Pak J Phytopathol 2008;19:86-8.

[17] Muley A, Lakhani J, Bhirud S, et al. Thrombocytopenia in plasmodium vivax malaria: how significant? Journal of Tropical Medicine 2014;2014:567469.
[18] Maina RN, Walsh D, Gaddy C, et al. Impact of Plasmodium falciparum infection on haematological parameters in children living in Western Kenya. Malaria Journal 2010;9(Suppl 3):S4.

[19] Adedapo AD, Falade CO, Kotila RT, et al. Age as a risk factor for thrombocytopenia and anemia in children treated for acute uncomplicated falciparum malaria. J Vector Borne Dis 2007;44(4):266-71.

[20] Lathia TB, Joshi R. Can hematological parameters discriminate malaria from non malarious acute febrile illness in the tropics? Indian J Med Sci 2004;58(6):23944.

[21] Kumar A, Shashirekha. Thrombocytopenia--an indicator of acute vivax malaria. Indian Journal of Pathology \& Microbiology 2006;49(4):505-8.

[22] George P, Alexander LM. A study on the clinical profile of complicated Plasmodium vivax mono-infections. Asian Pac J Trop Med 2010;3(7):560-2.

[23] Niazi GA. Haematological aspect of malaria in a population based hospital, Saudi Arabia. J Egypt Soc Parasitol 1995;25(3):787-93.

[24] Mert A, Ozaras R, Tabak F, et al. Malaria in Turkey: a review of 33 cases. Eur J Epidemiol 2003;18(6):579-82.

[25] Faseela TS, Roche RA, Anita KB, et al. Diagnostic value of platelet count in malaria. J Clin Diagn Res 2011;5(3):4646.

[26] Mohapatra MK, Padhiary KN, Mishra DP, et al. Atypical manifestations of Plasmodium vivax malaria. Indian Journal of Malariology 2002;39(1-2):18-25.

[27] Prasad R, Das BK, Pengoria R, et al. Coagulation status and platelet functions in children with severe falciparum malaria and their correlation of outcome. Journal of Tropical Pediatrics 2009;55(6):374-8.

[28] Murthy GL, Sahay RK, Srinivasan VR, et al. Clinical profile of falciparum malaria in a tertiary care hospital. J Indian Med Assoc 2000;98(4):160-2.

[29] Khan FY, Lutof AK, Yassin MA, et al. Imported malaria in Qatar: a one year hospital-based study in 2005. Travel Medicine and Infectious Disease 2009;7(2):111-7.

[30] González B, Rodulfo H, De Donato M, et al. Hematologic variations in patient with malaria caused by Plasmodium vivax before, during and after treatment. Invest Clin 2009;50(2):187-201.

[31] Fajardo LF, Tallent C. Malarial parasites within human platelets. JAMA 1974;229(9):1205-7.

[32] Makkar RPS, Mukhopadhyay S, Monga A, et al. Plasmodium vivax malaria presenting with severe thrombocytopenia. Braz J Infect Dis 2002;6(5):263-5. 